ICRR-Report 635-2012-24

IPMU12-0198

\title{
High Scale SUSY Breaking From Topological Inflation
}

\author{
Keisuke Harigaya $^{a}$, Masahiro Kawasaki ${ }^{b, a}$ and Tsutomu T. Yanagida ${ }^{a}$ \\ ${ }^{a}$ Kavli Institute for the Physics and Mathematics of the Universe, The University of \\ Tokyo, 5-1-5 Kashiwanoha, Kashiwa, Chiba 277-8583, Japan \\ ${ }^{b}$ Institute for Cosmic Ray Research, The University of Tokyo, 5-1-5 Kashiwanoha, \\ Kashiwa, Chiba 277-8582, Japan
}

\begin{abstract}
The recently observed mass $\sim 125 \mathrm{GeV}$ for the Higgs boson suggests a highenergy scale SUSY breaking, above $O(10) \mathrm{TeV}$. It is, however, very puzzling why nature chooses such a high energy scale for the SUSY breaking, if the SUSY is a solution to the hierarchy problem. We show that the pure gravity mediation provides us with a possible solution to this puzzle if the topological inflation is the last inflation in the early universe. We briefly discuss a chaotic inflation model in which a similar solution can be obtained.
\end{abstract}




\section{Introduction}

The ATLAS and CMS collaborations recently discovered a standard-model like Higgs boson of mass about $125 \mathrm{GeV}$ [1]. This observed Higgs mass, together with non-discovery of superpartners at LHC, suggests that the supersymmetry (SUSY) breaking scale is much higher than we expected, say above $O(10) \mathrm{TeV}$ [2]. However, if the SUSY is a solution to the hierarchy problem and hence its breaking is biased toward low energy scales, a crucial question naturally arises ; why does nature choose such a high energy scale for the SUSY breaking [3]? We show, in this letter, that the pure gravity mediation model recently proposed to explain the $125 \mathrm{GeV}$ Higgs mass [4] (for a similar model, see also [5]) provides us with a possible explanation for the high scale SUSY breaking if the topological inflation is the last inflation in the early universe.

It is believed that our universe experienced the quasi-exponential expansion called inflation [6] at its very early stage. Inflation makes our universe homogeneous and flat, which solves conceptual problems of big-bang cosmology, and also dilutes harmful relics like monopoles. Furthermore, quantum fluctuations of the inflaton $(=$ a scalar field that drives inflation) become classical by the cosmic expansion during inflation and result in density perturbations of the universe [7]. Inflation predicts nearly scale-invariant, adiabatic and gaussian density perturbations, which are consistent with the recent observations of the cosmic microwave radiation (CMB) [8]. Thus, the inflationary universe successfully describes our universe.

However, most inflation models have so called initial value problem [9], that is, they require tuning for the initial conditions of the inflaton and other relevant fields. Among many models, chaotic inflation [10] and topological inflation [11] are free from the initial value problem. It is well known that chaotic inflation occurs naturally from large field fluctuations at the Planck time. In topological inflation models, some discrete symmetry is spontaneously broken and topological defects (domain walls) are formed in the early universe. If the scalar field forming the defects has the vacuum expectation value larger than the Planck scale $\left(M_{\mathrm{pl}} \simeq 2.4 \times 10^{18} \mathrm{GeV}\right)$, the region inside the domain wall undergoes inflation. Since the defect formation is inevitable, inflation takes place naturally as long as the universe lives until the start of inflation. The longevity of the universe is not a problem in the open universe. Furthermore, open universes are likely created through tunneling in quantum cosmology [12].

We show, in this letter, that there is an upper bound of the reheating temperature,

$T_{R} \lesssim 10^{10} \mathrm{GeV}$, if the topological inflation takes place in the early universe. In the pure gravity mediation model, the wino is the LSP and it is the unique candidate of dark 
matter (DM) in the universe. The number density of the wino is almost proportional to the reheating temperature $T_{R}$ as long as its mass is lower than $1 \mathrm{TeV}$ [4]. We thus obtain a lower bound of the wino mass to explain the observed DM density as $m_{\text {wino }} \gtrsim 200 \mathrm{GeV}$. The lower bound of the wino mass is translated to the lower bound on the gravitino mass,

$m_{3 / 2} \gtrsim \mathcal{O}(10) \mathrm{TeV}$ in the pure gravity mediation model, implying scalar masses $\gtrsim \mathcal{O}(10)$ $\mathrm{TeV}$. Thus, there is a cosmological reason why the SUSY breaking scale is higher than $O(10) \mathrm{TeV}$ in the pure gravity mediation model if the topological inflation is the last inflation in our universe. We briefly note, in the last section, that a similar conclusion can be obtained in a chaotic inflation model.

\section{Topological Inflation Model}

It was pointed out long time ago [13, 14] that the topological inflation takes place for the following simple super potential and Kähler potential with $U(1)_{R} \times Z_{2}$ symmetry in the supergravity:

$$
\begin{aligned}
& W=v^{2} X\left(1-\sum_{n} \frac{g_{2 n}}{(2 n) !} \phi^{2 n}\right) \\
& K=|X|^{2}+|\phi|^{2}+k_{1}|X|^{2}|\phi|^{2}+\frac{k_{2}}{4}|X|^{4},
\end{aligned}
$$

where $g_{2 n}(n=1,2, .),. k_{1}$ and $k_{2}$ are coupling constant. We take a unit of the Planck scale $M_{\mathrm{pl}}=1$, here and hereafter. We have assumed that the expansions of super and Kähler potentials in the fields, $X$ and $\phi$, are well defined as long as the absolute values of the fields are within the Planck scale.

Thus, it is reasonable to consider all constants $g_{2 n}, k_{1}$ and $k_{2}$ are at most $O(1)$. For our analysis we neglect the higher order terms with $n \geq 2$ in the super potential, for simplicity. This may be valid as long as the expansion in $\phi$ converges sufficiently fast. In the followings, we assume this property about the expansion in the inflaton $\phi$. We have neglected possible higher order terms already in the Kähler potential in Eq. (2). We assume this model throughout this letter and consider that the main conclusion of this letter does not change even if we adopt another model for the topological inflation. $g \equiv g_{2}$ is chosen to be real and positive by a phase rotation of $\phi$.

$X$ and $\phi$ have $U(1)_{R}$ charges 2 and 0 , respectively. We also assume that $X$ is even and $\phi$ is odd under the $Z_{2}$, which is essential for the topological inflation to take place. The potential has a vacuum (see Eq. (1) with $n=1$ ),

$$
\langle X\rangle=0, \quad\langle\phi\rangle=\sqrt{\frac{2}{g}} .
$$


As shown in Ref. [14], the topological inflation takes place if $\langle\phi\rangle \gtrsim 1 / \sqrt{2}(g \lesssim 4)$. Since $\langle\phi\rangle \lesssim \mathcal{O}(1)$ for $g=\mathcal{O}(1)$, it is consistent with our assumption of neglecting higher order terms in the super and the Kähler potential.

The scalar potential derived from Eqs. (1) with $(n=1)$ and $(2)$ is, for $|X|$ and $|\phi| \ll 1$,

$$
\left.V=v^{4}\left|1-\frac{g}{2} \phi^{2}\right|^{2}\left[1+\left(1-k_{1}\right)|\phi|^{2}-k_{2}|X|^{2}\right)\right] .
$$

The $X$ field quickly settles down to the origin if $k_{2} \lesssim-1$, so hereafter we take $X=0$. We can identify the inflaton field as the real part of $\phi$. Using $\varphi=\sqrt{2} \operatorname{Re}(\phi)$, the potential is rewritten for $\varphi \ll 1$ as

$$
V \simeq v^{4}-\frac{1}{2}\left(g+k_{1}-1\right) v^{4} \varphi^{2} \equiv v^{4}-\frac{1}{2} \kappa v^{4} \varphi^{2}
$$

From this potential we obtain the $e$-folds $N$ as

$$
N=\int_{\varphi_{N}}^{\varphi_{f}} d \varphi \frac{V}{V^{\prime}} \simeq \frac{1}{\kappa} \ln \left(\frac{\varphi_{f}}{\varphi_{N}}\right),
$$

where $\varphi_{f}$ is the field value of $\varphi$ at the end of inflation. The slow roll parameters are given by

$$
\begin{aligned}
\epsilon & =\frac{1}{2} \frac{V^{\prime 2}}{V^{2}}=\frac{1}{2} \kappa^{2} \varphi^{2} \ll \eta \\
\eta & =\frac{V^{\prime \prime}}{V}=-\kappa
\end{aligned}
$$

For the inflation to produce the observed curvature perturbation, the inflaton potential satisfies $V^{3 / 2}\left(\varphi_{N}\right) / V^{\prime}\left(\varphi_{N}\right) \simeq 5 \times 10^{-4}$ for $N=50-60$, which leads to

$$
v \simeq 0.023 \sqrt{\kappa} e^{-\kappa N / 2} \simeq 10^{-3}
$$

for $\kappa=0.01$, where we have used $\varphi_{f} \simeq 1$. The inflaton mass is $m_{\phi} \simeq v^{2} \sqrt{2 g} \simeq 10^{13} \mathrm{GeV}$ for $g \simeq 1$.

The spectral index $n_{s}$ and tensor to scalar ratio $r$ are given by

$$
\begin{aligned}
n_{s} & =1-6 \epsilon+2 \eta=1-2 \kappa \\
r & =16 \epsilon=8 \kappa^{2} e^{-2 \kappa N} .
\end{aligned}
$$

Thus, $r$ is written as a function of $N_{s}$,

$$
r=2\left(1-n_{s}\right)^{2} \exp \left[-\left(1-n_{s}\right) N\right]
$$




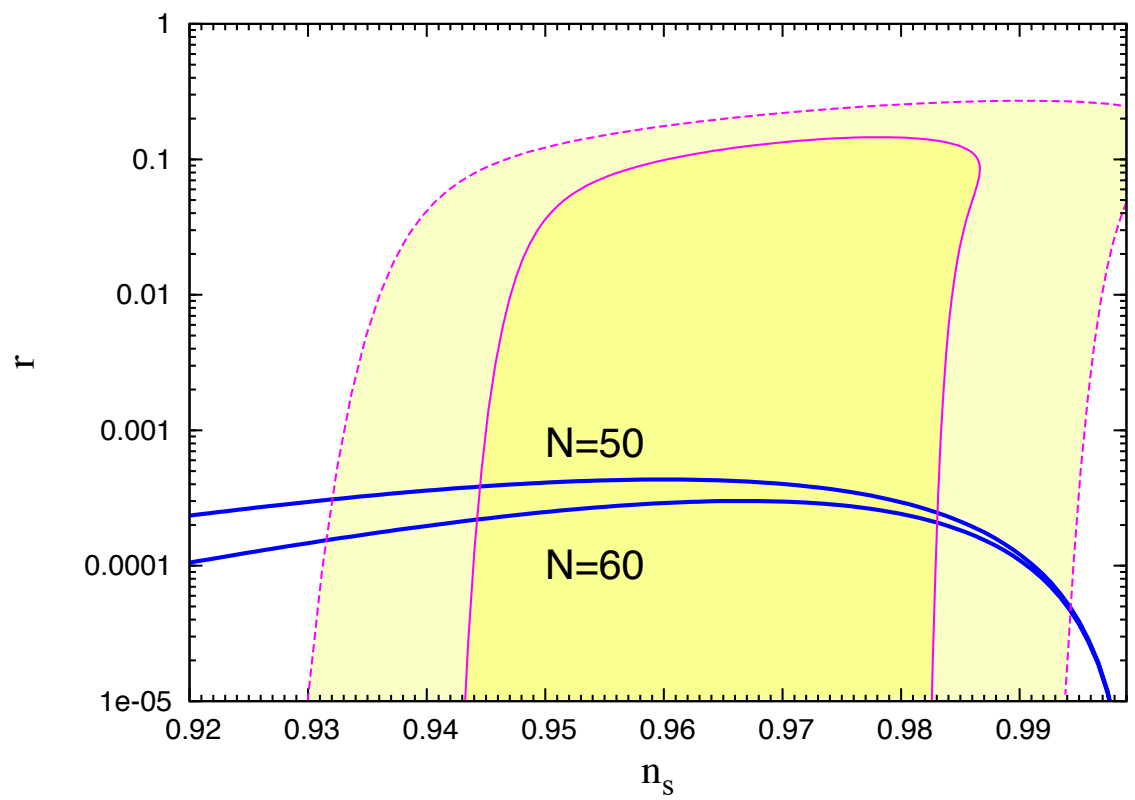

Figure 1: Prediction by the topological inflation in the $r-n_{s}$ plane. We also show the constraint from WMAP [8].

The prediction of the topological inflation is shown in Fig. 1 together with WMAP 7year constraint [8]. For the spectral index $n_{s} \simeq 0.94-0.98$ which is consistent with WMAP 7 year data, $r \simeq(3-4) \times 10^{-4}$. Therefore, the tensor mode that the topological inflation produces will be not detected even in the future satellite experiments such as CMBPol [15] and LiteBIRD [16].

We find that the value of the coupling constant $g$ is further restricted. If $g$ is too small, the vacuum $\langle\phi\rangle=\sqrt{2 / g}$ is far above one. On the other hands, a factor of $\exp \left(|\phi|^{2}\right)$ in the inflaton potential lifts up the potential above $\phi \simeq 1$. Therefore, the potential becomes a old-inflation type and the topological inflation fails. Now let us estimate the lower bound for $g$. The potential of the inflaton field is, for $X=0$,

$$
\begin{aligned}
V & =v^{4} \exp \left(\frac{\varphi^{2}}{2}\right)\left(1+\frac{k_{1}}{2} \varphi^{2}\right)^{-1}\left(1-\frac{g}{4} \varphi^{2}\right)^{2} \\
& \simeq v^{4} \exp \left(\frac{\varphi^{2}}{2}\right)\left(1+\frac{1-g}{2} \varphi^{2}\right)^{-1}\left(1-\frac{g}{4} \varphi^{2}\right)^{2}
\end{aligned}
$$

In the second line we have imposed $k_{1}+g \simeq 1$ which is required by the observation of the spectral index. The potential becomes a old-inflation type if there exists a point such that $\partial V / \partial \varphi=0$ for $0<\varphi<2 / \sqrt{g}$. By a simple algebraic calculation, we find that the 
condition \#1

$$
g>2-\sqrt{2} \simeq 0.59
$$

is required. On the other hand, if $\mathrm{g}$ is too large, $k_{1} \simeq 1-g$ is negatively large. From Eq. (13), one can see that the inflaton potential becomes singular before $\varphi$ reaches the vacuum. In order to avoid such behavior, the condition

$$
g<2
$$

is required. Eqs. (14) and (15) are consistent with our assumption that $g$ is of order one.

Now, let us estimate the reheating temperature after the inflation. There are four types of $R$ and $Z_{2}$ invariant interactions which contribute dominantly to the inflaton decay. One originates from the Kähler potential of the form

$$
K=\sum_{n+m=2} \frac{c_{n m}^{\prime \prime \prime}}{n ! m !} \phi^{n} \phi^{* m}|\Psi|^{2},
$$

where $\Psi$ is any field which is lighter than the half of the inflaton mass and $c_{n m}^{\prime \prime \prime}$ is a coupling constant of order one. However, with this type of interactions, the matrix element of the inflaton decay is proportional to square of the mass of $\Psi$ for any $n$ and $m$. Since the mass of $\Psi$ should be smaller than the half of the inflaton mass, decay width is suppressed in comparison with the mode described below at least by the factor of 16 . Therefore, we ignore the contribution from this type of interactions.

The other dominant interaction originates from the Kähler potential of the form

$$
K=c^{\prime \prime} \phi \phi^{*} H_{u} H_{d}
$$

where $H_{u}$ and $H_{d}$ are the up and down type Higgs field and $c^{\prime \prime}$ is the coupling constant of order one. This term is allowed since $H_{u} H_{d}$ has a vanishing $U(1)_{R}$ charge in the pure gravity mediation model in order to achieve an appropriate value for the $B \mu$ and $\mu$ term 4]. This Kähler potential leads to the inflaton interaction with the Higgs scalar as

$$
\mathcal{L}_{\text {int }}=c^{\prime \prime} \phi \partial_{\mu}\left(H_{u} H_{d}\right) \partial^{\mu} \phi^{*}+\text { h.c. }
$$

The decay rate of the inflaton to Higgs bosons is given by

$$
\Gamma_{\phi \rightarrow H_{u} H_{d}}=\frac{\left|c^{\prime \prime}\right|^{2}}{8 \pi}\langle\phi\rangle^{2} m_{\phi}^{3}
$$

\footnotetext{
${ }^{\# 1}$ Higher order terms in Kähler and super potentials may relax the constraints 14 and 15 .
} 
where we have used the inflaton mass $m_{\phi}=v^{2} \sqrt{2 g}$. The decay rate into Higgsinos is the same as this value.

There also exist interactions originate from the super potential. The small value of the $v^{2}$ required for the topological inflation in Eq. (9) is considered as a result of some new symmetry breaking, otherwise it should be $\mathcal{O}(1)$. For instance, consider a parity under which both of $X$ and $v^{2}$ transform as odd, then $v^{2} X$ is a completely neutral under all symmetries except for $U(1)_{R}$ (the $R$ charge of $X$ is two). The small value of $v$ is regarded as a small breaking of the parity. Thus, a term

$$
W=c^{\prime} v^{2} X H_{u} H_{d}
$$

is allowed in the super potential. This super potential leads to the inflaton interaction with the Higgs bosons as

$$
\mathcal{L}_{\text {int }}=\frac{g}{2} v^{4} \phi^{* 2} c^{\prime} H_{u} H_{d}+\text { h.c. }
$$

The decay rate due to this operator is given by

$$
\begin{aligned}
\Gamma_{\phi \rightarrow H_{u} H_{d}} & =\frac{\left|c^{\prime}\right|^{2}}{8 \pi} g^{2} \frac{v^{8}}{m_{\phi}}\langle\phi\rangle^{2} \\
& =\frac{\left|c^{\prime}\right|^{2}}{32 \pi} m_{\phi}^{3}\langle\phi\rangle^{2} .
\end{aligned}
$$

The last dominant interactions originate from the gauge kinetic functions:

$$
f=\frac{\delta_{a b}}{4}\left(1+\frac{c}{2} \phi^{2}\right) W_{\alpha}^{a} W_{\alpha}^{b}
$$

where $a, b, \cdots$ are the indices for gauge group and $c$ is a coupling constant of order one. This kinetic function leads to inflaton interactions with the gauge fields in the standard model as

$$
\mathcal{L}_{\text {int }}=-\frac{1}{4} \operatorname{Re}\left(\frac{c}{2} \phi^{2}\right) F_{\mu \nu}^{a} F^{a \mu \nu}+\frac{1}{8} \operatorname{Im}\left(\frac{c}{2} \phi^{2}\right) \epsilon^{\mu \nu \rho \sigma} F_{\mu \nu}^{a} F_{\rho \sigma}^{a},
$$

where $F_{\mu \nu}^{a}$ is the field strength of the gauge boson $A_{\mu}^{a}$, and Re and Im denote the real part and the imaginary part, respectively. Then the decay rate of the inflaton to the gauge fields is given by

$$
\Gamma_{\phi \rightarrow A^{a} A^{a}}=\frac{N_{A}|c|^{2}}{128 \pi}\langle\phi\rangle^{2} m_{\phi}^{3}
$$

$N_{A}=1+3+8=12$ is the number of the gauge fields. The contribution from the decay into gauginos is exactly the same one.

Adding above contributions together, the decay rate of the inflaton is given by

$$
\Gamma_{\phi}=\frac{8\left|c^{\prime \prime}\right|^{2}+\left|c^{\prime}\right|^{2}+6|c|^{2}}{32 \pi}\langle\phi\rangle^{2} m_{\phi}^{3}
$$




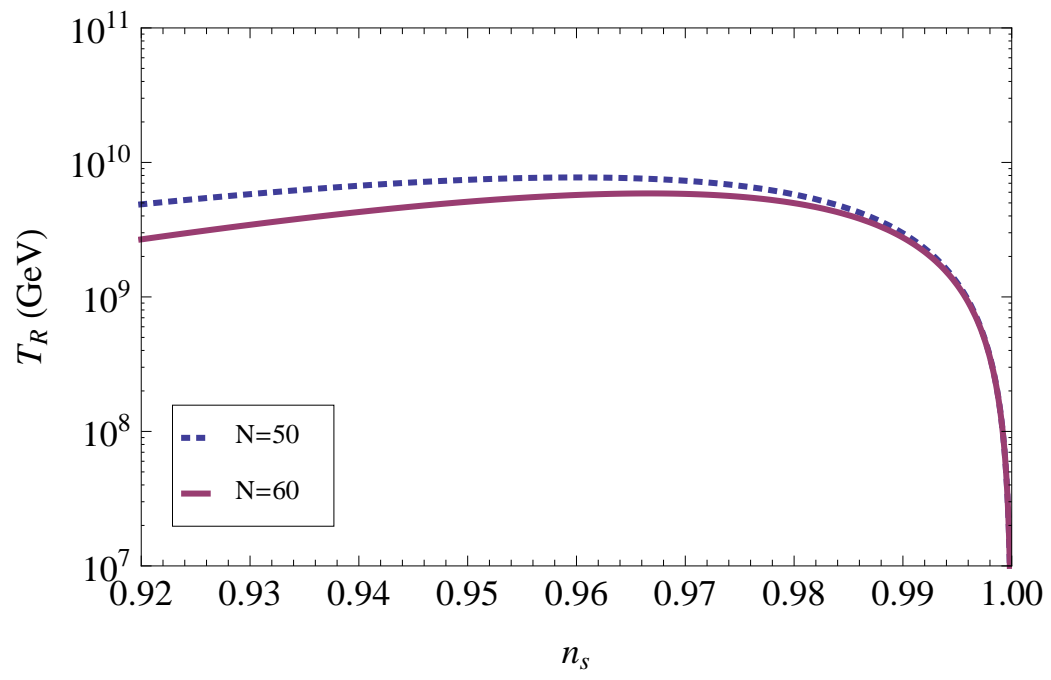

Figure 2: Reheating temperature for $N=50$ (upper curve) and 60(lower curve). We take $\left(\left(8\left|c^{\prime \prime}\right|^{2}+\left|c^{\prime}\right|^{2}+6|c|^{2}\right) / 15\right)^{\frac{1}{2}} g^{1 / 4}=1$.

From this decay rate we obtain the reheating temperature $T_{R}$ as

$$
T_{R} \simeq 0.25\left(\frac{8\left|c^{\prime \prime}\right|^{2}+\left|c^{\prime}\right|^{2}+6|c|^{2}}{15}\right)^{\frac{1}{2}} g^{-\frac{1}{2}} m_{\phi}^{3 / 2}=0.41\left(\frac{8\left|c^{\prime \prime}\right|^{2}+\left|c^{\prime}\right|^{2}+6|c|^{2}}{15}\right)^{\frac{1}{2}} g^{1 / 4} v^{3} .
$$

Using Eqs. (9) and (10), the reheating temperature is written as a function of the spectral index $n_{s}$,

$$
T_{R}=4.3 \times 10^{12} \mathrm{GeV}\left(\frac{8\left|c^{\prime \prime}\right|^{2}+\left|c^{\prime}\right|^{2}+6|c|^{2}}{15}\right)^{\frac{1}{2}} g^{1 / 4}\left(1-n_{s}\right)^{3 / 2} \exp \left[-\frac{3}{4} N\left(1-n_{s}\right)\right]
$$

which is shown in Fig. 2. We can see that $T_{R} \simeq(2-7) \times 10^{9} \mathrm{GeV}$ in the present model. This relatively low reheating temperature is a consequence of the $Z_{2}$ and the $U(1)_{R}$ symmetry, which is essential for the topological inflation. Taking into account $\mathcal{O}(1)$ ambiguity in the coupling constants $c$ 's, we safely conclude $T_{R} \lesssim 10^{10} \mathrm{GeV}$.

\section{Lower Bound on the Gravitino Mass in the Pure Gravity Mediation Model}

In the previous section, we have shown that the maximal reheating temperature is about $T_{R} \lesssim 10^{10} \mathrm{GeV}$ in a reasonable parameter range if the topological inflation is the last inflation in our universe. Now we are at the point to show that there is indeed a lower bound on the SUSY breaking energy scale, $m_{3 / 2} \gtrsim \mathcal{O}(10) \mathrm{TeV}$. 
First of all, the wino is the LSP and the unique candidate for the DM in the pure gravity mediation model [4]. The wino has a large annihilation cross section and hence the thermal wino can not provide a sufficient density for the observed DM as long as the mass of the wino $m_{\text {wino }} \lesssim 1 \mathrm{TeV}$. Thus, we must invoke a non thermal wino production in the early universe. The most promising process is the gravitino decay, since the gravitino is much heavier than the wino in the pure gravity mediation model.

Let us discuss the gravitino production in the high-energy thermal particle scattering in the early universe. Since the production of the gravitino occurs by Planck suppressed interactions, the production is more effective for higher temperatures. Therefore, the abundance of the gravitino is determined by the reheating temperature and given by [17]

$$
Y_{3 / 2} \equiv \frac{n_{3 / 2}}{s} \simeq 2.3 \times 10^{-12} \times \frac{T_{\mathrm{R}}}{10^{10} \mathrm{GeV}}
$$

Here, $n_{3 / 2}$ is the number density of the gravitino and $s$ is the entropy density of the universe.

The abundance of the wino from the decay of the gravitino is the same as Eq. (29), $n_{\text {wino }}=n_{3 / 2}$. After all, the energy fraction of the wino dark matter in the present universe $\Omega_{\text {wino }, 0}$ is given by $\# 2$

$$
\Omega_{\text {wino }, 0} \simeq \frac{m_{\text {wino }} Y_{3 / 2} s_{0}}{\rho_{\text {cr }, 0}} \simeq 0.12 h^{-2} \frac{m_{\text {wino }}}{200 \mathrm{GeV}} \frac{T_{\mathrm{R}}}{10^{10} \mathrm{GeV}}
$$

where $s_{0} \simeq 2.2 \times 10^{-11} \mathrm{eV}^{3}$ is the entropy density of the present universe, $\rho_{\mathrm{cr}, 0} \simeq 8.1 h^{-2} \times$ $10^{-11} \mathrm{eV}^{4}$ is the critical density of the present universe and $h$ is the scale factor for Hubble constant defined by $H_{0}=100 \mathrm{~h} \mathrm{~km} \mathrm{sec}^{-1} \mathrm{Mpc}^{-1}$.

The anthropic bound of the dark matter density is known as $\Omega_{\mathrm{DM}} h^{2} \gtrsim 0.1$ [18]. And as we have seen in the previous section, the reheating temperature $T_{\mathrm{R}}$ is bounded from above as $T_{\mathrm{R}} \lesssim 10^{10} \mathrm{GeV}$ if the topological inflation is the last inflation in the early universe. Therefore, we obtain the lower bound on the wino mass as

$$
m_{\text {wino }}>200 \mathrm{GeV}
$$

In the pure gravity mediation model, the wino mass is determined by the contribution from the anomaly mediation and the threshold correction by the Higgsino loop. Both contributions are of the same order and the wino mass has the upper bound as [4]

$$
m_{\text {wino }} \propto 10^{-2} m_{3 / 2}
$$

\footnotetext{
\#2 For $m_{\text {wino }} \gtrsim 1 \mathrm{TeV}$, the contribution from the thermally produced wino is not negligible. However, we can safely neglect this contribution in order to discuss the lower bound on the wino mass.
} 
Therefore, we obtain the lower bound for the SUSY breaking scale $m_{\mathrm{SUSY}}$;

$$
m_{\mathrm{SUSY}} \simeq m_{3 / 2}>\mathcal{O}(10) \mathrm{TeV} .
$$

\section{Conclusions and Discussion}

We have discussed the topological inflation in the previous section. However, as pointed out in the introduction, the chaotic inflation [10] is also interesting since there is no initial value problem. The chaotic inflation is easily constructed by using a shift symmetry in supergravity [19]. The Kähler potential is a function of $\phi+\phi^{\dagger}$ and the super potential is given by

$$
W=m X \phi,
$$

where $\phi$ is the inflaton. The inflaton $\phi$ can have an interaction in the Kähler potential,

$$
K=d\left(\phi+\phi^{\dagger}\right) H_{u} H_{d},
$$

where $d$ is a coupling constant of $O(1)$. It is easy to see that the upper bound of the reheating temperature $T_{R} \lesssim 10^{10} \mathrm{GeV}$ can be obtained for $d \lesssim O(1)$.

In this letter, we consider relatively high energy scales for the SUSY breaking. However, there is a very natural parameter region where the observed DM density is explained by a mixed wino-bino thermal relic DM of mass $\mathcal{O}(1) \mathrm{GeV}$ and sfermion masses are $\mathcal{O}(100)$ $\mathrm{GeV}$, even in the pure gravity mediation model [20]. However, this parameter region is excluded by too much non thermal DM production due to the overproduction of the gravitino in the inflaton decay [21].

We have assumed the $R$ parity conservation throughout this letter. However, if the $R$ parity is broken, the present argument is not applicable. The model which connects the SUSY breaking and the Peccei-Quinn symmetry breaking dynamics [20] may be interesting to understand the high scale SUSY breaking, if it is the case.

\section{Acknowledgments}

This work is supported by Grant-in-Aid for Scientific research from the Ministry of Education, Science, Sports, and Culture (MEXT), Japan, No. 14102004 (M.K.), No. 21111006 (M.K.), No. 22244021 (T.T.Y.), and also by World Premier International Research Center Initiative (WPI Initiative), MEXT, Japan. The work of K.H. is supported in part by a JSPS Research Fellowships for Young Scientists. 


\section{References}

[1] G. Aad et al. [ATLAS Collaboration], Phys. Lett. B 716, 1 (2012); S. Chatrchyan et al. [CMS Collaboration], Phys. Lett. B716, 30 (2012).

[2] Y. Okada, M. Yamaguchi and T. Yanagida, Prog. Theor. Phys. 85, 1 (1991); Y. Okada, M. Yamaguchi and T. Yanagida, Phys. Lett. B 262, 54 (1991); J. R. Ellis, G. Ridolfi and F. Zwirner, Phys. Lett. B 257, 83 (1991); H. E. Haber and R. Hempfling, Phys. Rev. Lett. 66, 1815 (1991).

[3] T. T. Yanagida and K. Yonekura, Phys. Lett. B 693, 281 (2010) arXiv:1006.2271 [hep-ph]]; F. Takahashi and T. T. Yanagida, Phys. Lett. B 698, 408 (2011) arXiv:1101.0867 [hep-ph]]; M. Bose and M. Dine, arXiv:1209.2488 [hep-ph]; B. Feldstein and T. T. Yanagida, arXiv:1210.7578 [hep-ph].

[4] M. Ibe and T. T. Yanagida, Phys. Lett. B 709, 374 (2012) arXiv:1112.2462 [hepph]]; M. Ibe, S. Matsumoto and T. T. Yanagida, Phys. Rev. D 85, 095011 (2012) arXiv:1202.2253 [hep-ph]]; B. Bhattacherjee, B. Feldstein, M. Ibe, S. Matsumoto and T. T. Yanagida, arXiv:1207.5453 [hep-ph].

[5] L. J. Hall, Y. Nomura and S. Shirai, arXiv:1210.2395 [hep-ph].

[6] A. H. Guth, Phys. Rev. D 23, 347 (1981); see also A. A. Starobinsky, Phys. Lett. B 91, 99 (1980); K. Sato, Mon. Not. Roy. Astron. Soc. 195, 467 (1981).

[7] V. F. Mukhanov and G. V. Chibisov, JETP Lett. 33, 532 (1981) [Pisma Zh. Eksp. Teor. Fiz. 33, 549 (1981)].

[8] E. Komatsu et al. [WMAP Collaboration], Astrophys. J. Suppl. 192, 18 (2011) arXiv:1001.4538 [astro-ph.CO]].

[9] A. D. Linde, Contemp. Concepts Phys. 5, 1 (1990) hep-th/0503203.

[10] A. D. Linde, Phys. Lett. B 129, 177 (1983).

[11] A. D. Linde, Phys. Lett. B 327, 208 (1994) astro-ph/9402031; A. Vilenkin, Phys. Rev. Lett. 72, 3137 (1994) hep-th/9402085.

[12] D. H. Coule and J. Martin, Phys. Rev. D 61, 063501 (2000) gr-qc/9905056; L. Susskind, In *Carr, Bernard (ed.): Universe or multiverse?* 247-266 th/0302219. 
[13] K. I. Izawa, M. Kawasaki and T. Yanagida, Prog. Theor. Phys. 101, 1129 (1999) hep-ph/9810537.

[14] M. Kawasaki, N. Sakai, M. Yamaguchi and T. Yanagida, Phys. Rev. D 62, 123507 (2000) hep-ph/0005073].

[15] D. Baumann et al. [CMBPol Study Team Collaboration], AIP Conf. Proc. 1141, 10 (2009) arXiv:0811.3919 [astro-ph]].

[16] LiteBIRD project, http://cmb.kek.jp/litebird/index.html.

[17] M. Kawasaki and T. Moroi, Prog. Theor. Phys. 93, 879 (1995) hep-ph/9403364, hep-ph/9403061; M. Kawasaki, K. Kohri, T. Moroi and A. Yotsuyanagi, Phys. Rev. D 78, 065011 (2008) arXiv:0804.3745 [hep-ph]].

[18] S. Hellerman and J. Walcher, Phys. Rev. D 72, 123520 (2005) hep-th/0508161; M. Tegmark, A. Aguirre, M. Rees and F. Wilczek, Phys. Rev. D 73, 023505 (2006) astro-ph/0511774.

[19] M. Kawasaki, M. Yamaguchi and T. Yanagida, Phys. Rev. Lett. 85, 3572 (2000) hep-ph/0004243.

[20] B. Feldstein and T. T. Yanagida in [3].

[21] M. Endo, F. Takahashi and T. T. Yanagida, Phys. Rev. D 76, 083509 (2007) arXiv:0706.0986 [hep-ph]]. 